\title{
Raman scattering from heavily doped (311) GaAs:Si grown by molecular beam epitaxy
}

\author{
S. H. Kwok and R. Merlin \\ The Harrison M. Randall Laboratory of Physics, The University of Michigan, Ann Arbor, \\ Michigan 48109-1120 \\ W. Q. Li and P. K. Bhattacharya \\ Solid State Electronics Laboratory, Department of Electrical Engineering and Computer Science, \\ The University of Michigan, Ann Arbor, Michigan 48109-2122
}

(Received 2 December 1991; accepted for publication 25 March 1992)

\begin{abstract}
Raman scattering by localized vibrational modes and plasmons has been used to characterize heavily $p$ - and $n$-type silicon-doped (311) $A$ GaAs layers grown by molecular beam epitaxy. Consistent with the doping character, $p$-type samples show two modes associated with $\mathrm{Si}(\mathrm{As})$ and the complex defect $\mathrm{Si}-\mathrm{X}$. Acceptor-related lines were not observed in $n$-type samples, an indication that compensation levels in the layers are very low. The results are discussed in relation to growth conditions on (311) $A$ surfaces.
\end{abstract}

Silicon is the most widely used $n$-type dopant in GaAs and other III-V compound semiconductors grown by molecular beam epitaxy (MBE). Because Si is an amphoteric impurity, however, its doping behavior depends on various factors including the substrate morphology, surface bonding configuration, growth temperatures, and the As/Ga flux ratio. Recently, there have been several studies of MBE Si-doped $\mathrm{GaAs}$ grown on substrates of different orientations. ${ }^{1,2}$ To effectively correlate transport properties with growth conditions, it is important to characterize the distribution of $\mathrm{Si}$ among the As and Ga sublattices. Raman and infrared (IR) spectroscopy, probing local vibration modes (LVM), are the most widely used techniques for this purpose. Because of various reasons, the former technique exhibits many advantages over IR in the case of thin-film structures. Recently, Raman scattering has been applied to the study of LVM in heavily Si-doped GaAs prepared by ion implantation followed by thermal annealing, ${ }^{3-5}$ liquid-phase epitaxy, ${ }^{6}$ and $\mathrm{MBE}^{3,7}$ In this work, we present Raman results on Si-doped GaAs grown on (311) $A$ GaAs substrates by MBE. The (311) $A$ surface is particularly interesting because the densities of $\mathrm{Ga}$ single-bonding and As double-bonding sites are equal. Accordingly, Si could either incorporate into the Ga sublattice and, so, act as a donor or into the As sites as a $p$-type dopant, depending on the growth conditions. This feature is expected to be of importance in device applications provided high levels of both types of doping could be obtained in a reproducible manner.

Si-doped GaAs layers ( $1 \mu \mathrm{m}$ thick) were grown by MBE on $n$-type (311) $A$ GaAs substrates. Here, data are reported for three samples. Sample A was grown at a substrate temperature of $670^{\circ} \mathrm{C}$ with flux ratio $\mathrm{As} / \mathrm{Ga} \sim 1$ while the substrate temperature for sample B was $450^{\circ} \mathrm{C}$ and the flux ratio was $\mathrm{As} / \mathrm{Ga}>4$. The growth rates in both cases were kept at $0.5 \mu \mathrm{m} / \mathrm{h}$. The as-grown samples were measured using standard capacitance-voltage $(C-V)$ techniques to obtain doping types and carrier concentrations. Sample A shows $p$-type conductivity with a free hole concentration of $2.1 \times 10^{19} \mathrm{~cm}^{-3}$ and sample $B$ is $n$-type with a free electron concentration of $1.2 \times 10^{19} \mathrm{~cm}^{-3}$. Sample C is an undoped sample grown for reference purposes. We notice that the doping level in sample $A$ is the highest ever obtained for $p$-type doping in GaAs.

Raman data were obtained with a Dilor $X Y$ spectrometer. Spectra were recorded at room temperature in the backscattering configuration with the incident and scattered light polarized either along the $x=(0 \overline{1} 1)$ or $y$ $=(\overline{2} 33)$ directions. The 4765 and $4965 \AA$ lines of an $\mathrm{Ar}^{+}$ laser were used as excitation sources. The penetration depth for such wavelengths is considerably smaller than the thicknesses of the layers.

In (311) backscattering, both the LO (longitudinaloptic) and TO (transverse-optic) modes are allowed. In heavily doped GaAs, however, the LO phonon couples to the electron (or hole) plasmon giving rise to two coupled modes, $L^{+}$and $L^{-8}{ }^{8}$ Figure 1 shows the Raman spectrum of the $n$-type film at large energy shifts. The relatively broad feature with maximum at $\sim 1080 \mathrm{~cm}^{-1}$ is the $L^{+}$ plasmon-like mode. Its frequency corresponds ${ }^{9}$ to a free electron concentration of $(1.2 \pm 0.2) \times 10^{19} \mathrm{~cm}^{-3}$, in very good agreement with the $C-V$ measurements.

The low frequency regions of the spectra of the p-type (sample A), $n$-type (sample B) and undoped (sample C) films are shown in Fig. 2. The weak peak at $\sim 340 \mathrm{~cm}^{-1}$ and the structures between 450 and $600 \mathrm{~cm}^{-1}$ in all samples are due to second-order phonon scattering (small differences among the spectra are due to slight misalignments in the scattering geometry). Other than the intrinsic modes, the $p$-type sample shows additional features at 365 and $394 \mathrm{~cm}^{-1}$, which are ascribed to $\mathrm{Si}-\mathrm{X}$ and $\mathrm{Si}(\mathrm{As})$ LVM, respectively. ${ }^{7} \mathrm{Si}(\mathrm{As})$ denotes the local vibration associated with a $\mathrm{Si}$ atom replacing $\mathrm{As}$ while $\mathrm{Si}-\mathrm{X}$ is a controversial defect complex. ${ }^{7,10}$ Murray et $a l^{7}$ found that the Si-X defect can be best explained as a nearest-neighbor double or triple acceptor $\mathrm{Si}(\mathrm{As})-V(\mathrm{Ga})$ pair, where $V(\mathrm{Ga})$ denotes a vacant $\mathrm{Ga}$ sublattice site. Of course, $\mathrm{Si}(\mathrm{As})$ is a single acceptor. The fact that the $n$-type sample does not show features associated with acceptor sites indicates that levels of compensation are low for our structures. However, the sample does not show evidence of $\mathrm{Si}(\mathrm{Ga})$ either. But, it is known that such a line can only be 


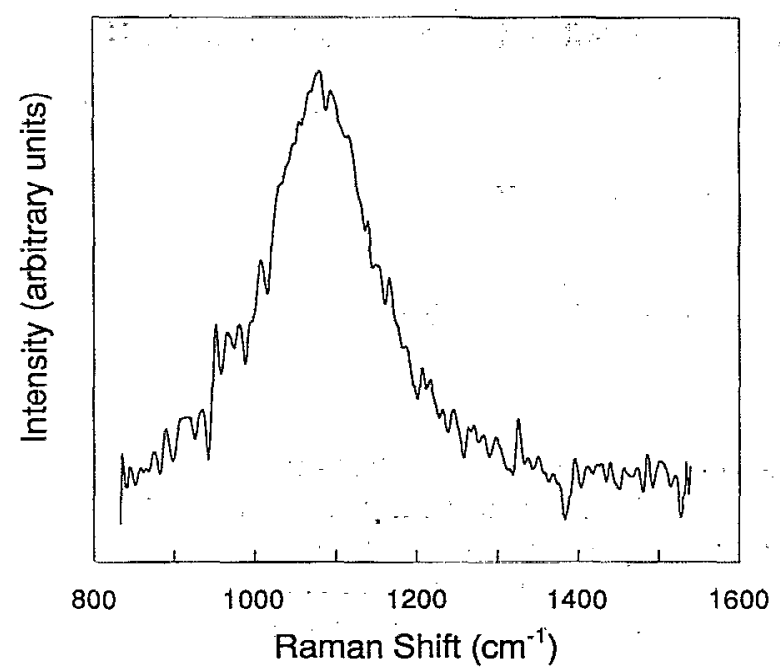

FIG. 1. Raman scattcring from the $n$-type film (samplc A) showing the $L^{+}$plasmon-like mode. The scattering configuration is $z(x, x) \bar{z}$ and the excitation wavelength is $4765 \AA(T=300 \mathrm{~K})$.

observed in the immediate vicinity of $E_{1}$ resonance at $\sim 3$ $\mathrm{eV}^{3}$

A qualitative account of our findings is discussed in the following. As shown in Fig. 3, there are equal density of single dangling-bond sites ( $\mathrm{Ga}$ ) and double dangling-bond sites (As) at the (311)A surface. Si behaves as a donor in $\mathrm{Ga}$ sites while it acts like an acceptor in As sites. The actual distribution of $\mathrm{Si}$ atoms depends not only on the relative strengths of the $\mathrm{Si}-\mathrm{Ga}$ and $\mathrm{Si}-\mathrm{As}$ bonds, but also on the fact that during growth $\mathrm{Si}$ must"compete with As and Ga to occupy the single-bond acceptor sites and doublebond donor sites. It appears that, at high temperatures and low As-fluxes, molecular $\mathrm{As}_{4}$ is a much less effective competitor of $\mathrm{Si}$ at single-bond acceptor sites. This is likely



FIG. 2. Raman spectra of the $p$-type (top), $n$-type (middle), and undoped (bottom) (311) samples. The local vibrational modes of the $p$-type sample are labeled by arrows. The scattering configuration is $z(y, y) \bar{z}$ and the excitation wavelength is $4965 \AA(T=300 \mathrm{~K})$.

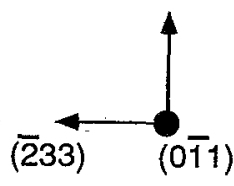

As

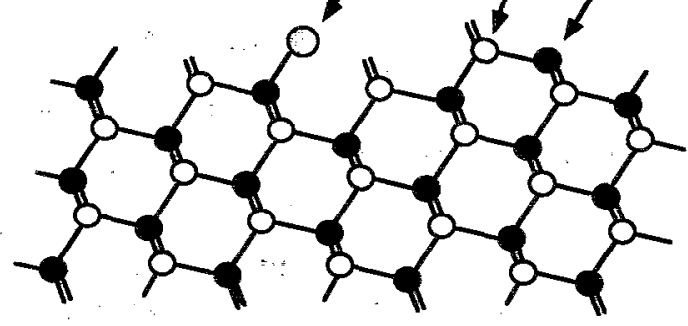

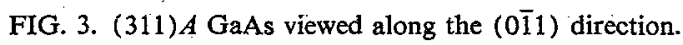

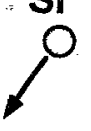

because the sticking coefficient of As decreases with increasing temperature. Moreover, the molecular $\mathrm{As}_{4}$ must be broken up, the energy for which is less readily provided by forming only a single-bond per atom as opposed to two bonds on (100) surfaces. These circumstances, together with the competition from plentiful $\mathrm{Ga}$ atoms at the double-donor sites, forming two strong Ga-As bonds, should effectively shift Si atoms to the acceptor sites on the As sublattices (as seen in sample A), even though the $\mathrm{Si}$ atom itself would bind more strongly on the donor sites. With lower temperatures and increasing As/Ga flux ratios, the balance should shift back in the direction of the energetically preferred $\mathrm{Ga}$ sites (as seen in sample B), leading to $n$-type doping.

In conclusion, we have observed Raman scattering by local vibrational modes in MBE (311) p-type GaAs which is consistent with the character of the sample. $\mathrm{Si}$ acts like an acceptor by either occupying As sites or by forming $\mathrm{Si}-\mathrm{X}$ complexes. The $n$-type sample does not show any acceptor feature indicating weak levels of compensation. These results are extremely encouraging and suggest that devices such as high-frequency bipolar transistors can be grown on $311(A)$ GaAs by all-silicon doping.

This work was supported by the Army Research Office (URI program) under Contract No. DAAL03-86-K0007 and the University Research Initiative Program (Contract No. AFOSR-90-0214).

${ }^{1}$ W. I. Wang, E. E. Mendez, T. S. Kuen, and L. Esaki, Appl. Phys. Lett. 47,826 (1985).

${ }^{2}$ W. Q. Li and P. K. Bhattacharya, IEEE Elcetron Device Lett. 13, 29 (1992).

${ }^{3}$ M. Ramsteiner, J. Wagner, H. Ennen, and M. Maier, Phys. Rev. B.38, 10669 (1988).

${ }^{4}$ T. Nakamura and T. Katoda, J. Appl. Phys. 57, 1084 (1985):

${ }^{5}$ M. Holtz, R. Zallen, A. E. Geissberge, and R. A. Sadler, J. Appl. Phys. 59, 1946 (1986).

${ }^{6}$ T. Kamijoh, A. Hashimoto, H. Tokano, and M. Sukata, J. Appl. Phys. 59, $2382(1986)$.

${ }^{7}$ R. Murray, R. C. Newman, M. J. L. Songster, R. B. Beall, J. J. Hanis, P. J. Wright, J. Wagner, and M. Ramsteiner, J. Appl. Phys. 66, 2589 (1989):

${ }^{8} \mathrm{M}$. V. Klein, in Light Scattering, in Solids $I$, edited by M. Cardona (Springer, New York, 1975), Chap. 4.

${ }^{9}$ H. Yao and A. Compaan, Appl. Phys. Lett. 57, 147 (1990).

${ }^{10}$ M. Yematsu and K. Maezawa, Jpn. J. Appl. Phys. 29, L527 (1990). 